\title{
Reconfigurable Intelligent Surface Aided NOMA Networks
}

\author{
Tianwei Hou, Student Member, IEEE, Yuanwei Liu, Senior Member, IEEE, Zhengyu Song*, Xin Sun, Yue \\ Chen, Senior Member, IEEE, and Lajos Hanzo*, Fellow, IEEE
}

\begin{abstract}
Reconfigurable intelligent surfaces (RISs) constitute a promising performance enhancement for next-generation (NG) wireless networks in terms of enhancing both their spectral efficiency (SE) and energy efficiency (EE). We conceive a system for serving paired power-domain non-orthogonal multiple access (NOMA) users by designing the passive beamforming weights at the RISs. In an effort to evaluate the network performance, we first derive the best-case and worst-case of new channel statistics for characterizing the effective channel gains. Then, we derive the best-case and worst-case of our closed-form expressions derived both for the outage probability and for the ergodic rate of the prioritized user. For gleaning further insights, we investigate both the diversity orders of the outage probability and the high-signalto-noise (SNR) slopes of the ergodic rate. We also derive both the SE and EE of the proposed network. Our analytical results demonstrate that the base station (BS)-user links have almost no impact on the diversity orders attained when the number of RISs is high enough. Numerical results are provided for confirming that: i) the high-SNR slope of the RIS-aided network is one; ii) the proposed RIS-aided NOMA network has superior network performance compared to its orthogonal counterpart.
\end{abstract}

Index Terms-NOMA, passive beamforming, reconfigurable intelligent surface.

\section{INTRODUCTION}

The demand for next-generation (NG) networks having high energy efficiency (EE) has been rapidly increasing [2]. A variety of sophisticated wireless technologies have been proposed for NG networks, including massive multiple-input multiple-output (MIMO) and millimeter wave (mmWave) communications [3]. Recently, cost-efficient reconfigurable intelligent surfaces (RISs) have been proposed for cooperative NG networks [4-6].

To enhance both the spectral efficiency (SE) and EE of NG networks, non-orthogonal multiple access (NOMA) has been

Manuscript received December 20, 2019; revised April 28, 2020; accepted May 9, 2020. Date of publication ; date of current version

L. Hanzo would like to acknowledge the financial support of the Engineering and Physical Sciences Research Council projects EP/N004558/1, EP/P034284/1, EP/P034284/1, EP/P003990/1 (COALESCE), of the Royal Society's Global Challenges Research Fund Grant as well as of the European Research Council's Advanced Fellow Grant QuantCom. This paper is also supported by the National Natural Science Foundation of China under Grant 61901027. This paper was presented at the IEEE Global Communication Conference, Taipei, China, Dec. 2020 [1]. (Corresponding authors: Zhengyu Song and Lajos Hanzo.)

T. Hou, Z. Song and X. Sun are with the School of Electronic and Information Engineering, Beijing Jiaotong University, Beijing 100044, China (email: 16111019@bjtu.edu.cn, songzy@bjtu.edu.cn, xsun@bjtu.edu.cn).

Y. Liu and Yue Chen are with School of Electronic Engineering and Computer Science, Queen Mary University of London, London E1 4NS, U.K. (e-mail: yuanwei.liu@qmul.ac.uk, yue.chen@qmul.ac.uk).

L. Hanzo is with University of Southampton, Southampton, U.K. (email:1h@ecs.soton.ac.uk). proposed as a promising technique of opportunistically capitalizing on the users' specific channel state information (CSI) differences [7-9]. NOMA networks are capable of serving multiple users at different quality-of-service (QoS) requirements in the same time/frequency/code resource block [1012]. Hence, inspired by the aforementioned benefits of NOMA and RIS techniques, we explore the network's performance enhanced by the intrinsic integration of the power-domain NOMA $^{1}$ and RIS techniques in the downlink (DL).

\section{A. Prior Work}

In recent years, RIS based techniques have received considerable attention owing to their beneficial applications [13-15]. The RIS aided system comprises an array of intelligent surface units, each of which can independently absorb energy and shift the phase of the incident signal. By appropriately adjusting the reflection angles and amplitude coefficients of RIS elements, the electromagnetic signal can be reconfigured for wireless transmission. The performance of RIS-aided and relay-assisted networks was compared in [16], indicating that RIS-aided networks may have better network performances, provided that the number of RISs is high enough. The associated energy consumption model was proposed in $[17,18]$, where the EE of the proposed network was optimized. Numerous application scenarios, such as RISs aided physical layer security relying on cooperative jamming techniques have also been considered $[19,20]$. The RIS components are capable of blocking the signal of eavesdroppers, hence enhancing the secrecy performance. RIS assisted simultaneous wireless information and power transfer (SWIPT) was proposed in [21] for the users located in coverage-holes. In the 5G new radio (NR) standard, the coverage area is significantly reduced for carriers beyond $6 \mathrm{GHz}$ [22]. Hence, a sophisticated signal alignment strategy was employed at the RISs for coverage area enhancement in mmWave scenarios [23]. However, in most previous research, continuous amplitude coefficients and phase shifts were assumed at the RISs [24], whilst in practice the phase shifts of RISs may not be continuous. Thus discrete phase shifts were considered in [25] for a multiple-input single-output (MISO) assisted RIS network. The channel capacity of a RISaided MIMO network was maximized, where both analog and digital beamforming, as well as hybrid beamforming were considered [26]. Furthermore, focusing on the user's fairness,

\footnotetext{
${ }^{1}$ In this article, we use NOMA to refer to power-domain NOMA for
} simplicity. 
a fairness-oriented design (FOD) was proposed in a RIS-aided MIMO network [27].

To further enhance both the SE and EE of the DL, NOMA and RIS techniques were integrated in [28]. The RISs can be deployed for enhancing the power level of the cell-edge users, where the cell-center users treat the reflected signal as interference [28]. Both continuous and discrete phase shifters were used in a RIS-aided MISO NOMA network [29]. Naturally, the BS-user link plays a key role [30]. A RIS-aided NOMA network was also investigated in [31], whilst the BSuser link and the BS-RIS link, as well as the RIS-user link were assumed to experience Rayleigh fading. The associated bit error ratio (BER) was evaluated in the case of Rayleigh fading in [32]. However, both the BS and RISs are part of the infrastructure, and the RISs are typically positioned for exploiting the line-of-sight (LoS) path with respect to the fixed BS in NG networks for increasing the received signal power. Hence, the impact of fading environments on RIS networks has also attracted attention [33]. A fairness-oriented algorithm was proposed in a RIS-aided NOMA network [33], where Rician fading channels were used for modelling the channel gains. Note that when the Nakagami and Rice fading parameter obey the following constraint $m=\frac{(K+1)^{2}}{2 K+1}$, these fading channels are identical [34, eq. (3.38)].

\section{B. Motivations and Contributions}

The above-mentioned papers mainly studied the network's fairness, whilst there is a paucity of investigations on the SE improvement of NOMA networks. To comprehensively analyze the network's performance enhanced by RISs, a RISaided SISO-NOMA network is proposed. Motivated by the potential joint benefits of RISs and NOMA networks, whilst relying on analog beamforming [35], in this article we will analyse the performance of a RIS-aided NOMA DL scenario, where a priority-oriented design (POD) is proposed, which is also capable of enhancing the SE. In the proposed POD, we improve the performance of the user having the best channel gain, where all other users rely on RIS-aided beamforming. In contrast to previous contributions [27], we will show that the proposed POD outperforms the FOD in terms of its SE.

Against to above background, our contributions can be summarized as follows:

- We propose a novel RIS-aided NOMA network, where a POD is employed for enhancing the SE. The impact of the LoS transmission on the reflected BS-RIS-user links are exploited. Furthermore, the impact of the proposed design on the attainable performance is characterized in terms of its outage probability (OP), ergodic rate, SE and EE.

- Explicitly, we derive closed-form expressions of both the OP and of the ergodic rate for the proposed RISaided NOMA network. Both the best-case and worstcase of the OP and of the ergodic rate are derived. Both accurate and approximate closed-form expressions are derived. Furthermore, both the diversity orders and highSNR slopes are obtained based on the OP and ergodic
TABLE I:

TABLE OF NOTATIONS

\begin{tabular}{|l|r|}
\hline$R_{W}$ & The target rate of user $W$. \\
\hline$m_{1}$ & Fading parameter between the BS and RISs. \\
\hline$m_{W}$ & Fading parameter between the RISs and user $W$. \\
\hline$W$ & The number of users. \\
\hline$N$ & The number of RISs. \\
\hline $\mathbf{h}$ & The channel vector between the BS and RISs. \\
\hline $\mathbf{g}_{W}$ & The channel vector between the RISs and user $W$. \\
\hline$r_{W}$ & The channel gain between the BSs and user $W$. \\
\hline $\mathbf{\Phi}$ & The effective matrix of RISs. \\
\hline
\end{tabular}

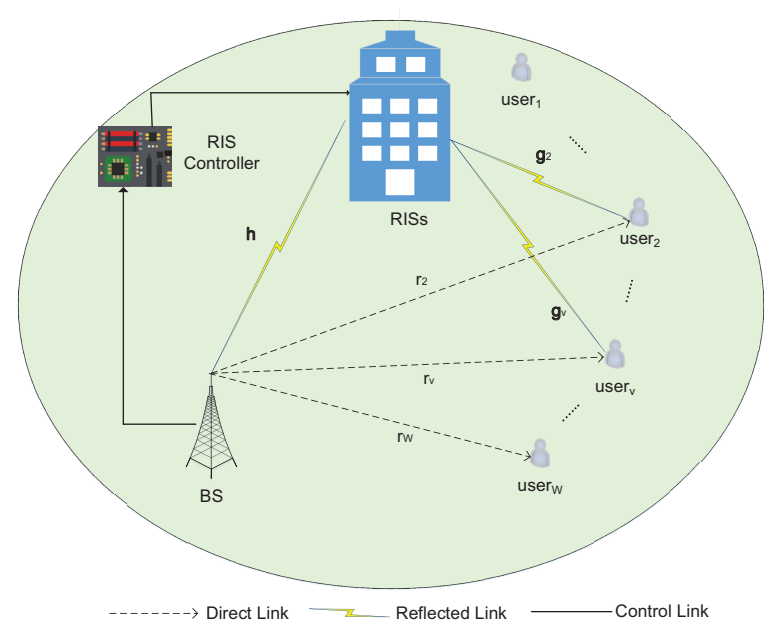

Fig. 1: Illustration of RIS-aided NOMA networks.

rate. The results confirm that the diversity order can be enhanced by increasing the number of RISs.

- The simulation results confirm our analysis, illustrating that: 1) the BS-user link can be ignored when the number of RISs is high enough; 2) the RIS-aided NOMA network relying on the optimal power allocation factors is capable of outperforming its OMA counterpart; 3) the SE of the proposed POD can be significantly enhanced compared to the FOD, when the number of RISs is high enough.

\section{Organization and Notations}

The rest of the paper is organized as follows. In Section II, the model of RIS-aided NOMA networks is discussed. Our analytical results are presented in Section III, while our numerical results are provided in Section IV for verifying our analysis, followed by our conclusions in Section V. The distribution of a random variable with mean $x$ and covariance matrix $k$ is denoted by $\mathcal{R} \mathcal{V}(x, k)$; and $\sim$ stands for "distributed as". $\mathbb{P}(\cdot)$ and $\mathbb{E}(\cdot)$ represent the probability and expectation, respectively. Table I lists the main notations used in this article.

\section{SySTEM MODEL}

Let us consider the RIS-aided NOMA DL, where a BS equipped with a single transmit antenna (TA) is communicating with $W$ users, each equipped with a single receive (RA) antenna. We have $N>1$ intelligent surfaces at the 
appropriate location. By appropriately adjusting the reflection angles and amplitude coefficients of the RIS elements, the electromagnetic signal can be beneficially manipulated. Fig. 1 illustrates the wireless communication model for a single BS.

\section{A. RIS-Aided SISO-NOMA Network}

We first provide a fundamental model to illustrate the network performance affected by RISs. In order to illustrate the LoS links between the BS and RISs, the small-scale fading vector is defined as

$$
\mathbf{h}=\left[\begin{array}{c}
h_{1} \\
\vdots \\
h_{N}
\end{array}\right]
$$

where $\mathbf{h}$ is a $(N \times 1)$-element vector whose elements represent the Nakagami fading channel gains. The probability density function (PDF) of the elements can be expressed as

$$
f_{1}(x)=\frac{m_{1}^{m_{1}} x^{m_{1}-1}}{\Gamma\left(m_{1}\right)} e^{-m_{1} x}
$$

where $m_{1}$ denotes the fading parameter, and $\Gamma(\cdot)$ represents the Gamma function. Note that $\Gamma\left(m_{1}\right)=\left(m_{1}-1\right)$ ! when $m_{1}$ is an integer.

It is assumed that there are a total of $W$ users in the cluster, where the pair of users, user $W$ and user $v$ with $1 \leq v<W$, are superimposed for DL transmission in NOMA. Hence, the small-scale fading vector between the RISs and user $W$ is defined as

$$
\mathbf{g}_{W}=\left[\begin{array}{lll}
g_{W, 1} & \cdots & g_{W, N}
\end{array}\right] .
$$

Similarly, the small-scale fading vector between the RISs and user $v$ is given by

$$
\mathbf{g}_{v}=\left[\begin{array}{lll}
g_{v, 1} & \cdots & g_{v, N}
\end{array}\right],
$$

where $\mathbf{g}_{W}$ and $\mathbf{g}_{v}$ are $(1 \times N)$-element vectors whose elements represent the Nakagami fading channel gains having fading parameters of $m_{W}$ and $m_{v}$, respectively.

Due to the strong scattering environment, the BS-user link between the BS and user $W$ as well as that between the BS and user $v$ are modelled by Rayleigh fading, which can be expressed as $r_{W}$ and $r_{v}$, respectively.

In DL transmission, the BS sends the following signal to the paired NOMA users:

$$
\mathbf{s}=\alpha_{v}^{2} \mathbf{s}_{v}+\alpha_{W}^{2} \mathbf{s}_{W}
$$

where $\mathbf{s}_{v}$ and $\mathbf{s}_{W}$ denote the signal intended for user $v$ and user $W$, respectively, with $\alpha_{v}^{2}$ and $\alpha_{W}^{2}$ representing the power allocation factors of user $v$ and user $W$, respectively. Based on the NOMA protocol, we have $\alpha_{v}^{2}+\alpha_{W}^{2}=1$.

RIS networks are typically classified into two categories [36], i.e. anomalous reflectors or diffuse scatterers for mmWave and sub-6G networks, respectively. When the size of RISs is comparable to the wavelength for the sub-6G networks, they may be modelled as diffusers. In radar networks, the path loss of the BS-RIS and RIS-user links are expected to obey the product-distance law [37]. By contrast, when the size of the RISs is sufficiently large compared with the wavelength, the generalized Snell law as well as the theory of geometric optics are applicable to model the path loss [37]. Without loss of generality, we focus our attention on user $W$ in sub-6G networks, when the signal received by user $W$ from the BS through RISs is given by [36]

$$
y_{w}=\left(\mathbf{g}_{w} \mathbf{\Phi} \mathbf{h} d_{1}^{-\frac{\alpha_{l}}{2}} d_{2, w}^{-\frac{\alpha_{l}}{2}}+r_{w} d_{3, w}^{-\frac{\alpha_{n}}{2}}\right) p \mathbf{s}+N_{0}
$$

where $p$ denotes the transmit power of the BS, $\boldsymbol{\Phi} \triangleq$ $\operatorname{diag}\left[\beta_{1} \phi_{1}, \beta_{2} \phi_{2}, \cdots, \beta_{N} \phi_{N}\right]$ is a diagonal matrix, which accounts for the effective phase shift applied by all intelligent surfaces, $\beta_{n} \in(0,1]$ represents the amplitude reflection coefficient of RISs, while $\phi_{n}=\exp \left(j \theta_{n}\right), j=\sqrt{-1}, \forall n=$ $1,2 \cdots, N$, and $\theta_{n} \in[0,2 \pi)$ denotes the phase shift introduced by the $n$-th intelligent surface. Based on the insights from [38], the amplitude coefficient of each RIS elements can be different, but in order to obtain tractable analytical results, we set $\beta_{1}, \ldots, \beta_{N}=1$ in Problem (9) for simplicity. It is assumed that the CSIs are perfectly known at the RIS controller [25]. Still referring to (6), $d_{1}$ and $d_{2, w}$ denote the distance between the BS and RISs as well as that between the RISs and user $w$, while $d_{3, w}$ denotes the distance between the BS and user $w^{2}$. Furthermore, $\alpha_{l}$ and $\alpha_{n}$ denote the path loss exponent of the BS-RIS-user links and BS-user links. Finally, $N_{0}$ denotes the additive white Gaussian noise (AWGN), which is modeled as a realization of a zero-mean complex circularly symmetric Gaussian variable with variance $\sigma^{2}$.

\section{RIS DESIGN FOR THE PRIORITIZED USER IN NOMA NETWORKS}

In this section, we first design the phase shifts and reflection amplitude coefficients for the RISs. Our new channel statistics, OPs, ergodic rates, SE and EE are illustrated in the following subsections.

\section{A. RIS Design}

When the direct BS-user signal and reflected BS-RIS-user signals are co-phased, the channel gain of user $W$ is given by

$$
\left|\tilde{h}_{W}\right|^{2}=\left|\mathbf{g}_{W} \boldsymbol{\Phi} \mathbf{h} d_{1}^{-\frac{\alpha_{l}}{2}} d_{2, W}^{-\frac{\alpha_{l}}{2}}+r_{W} d_{3, W}^{-\frac{\alpha_{n}}{2}}\right|^{2} .
$$

It is assumed that there are $W$ users in the cluster, and then the achievable channel gains of users $1 \cdots W$ are ordered as follows [40]:

$$
\left|\tilde{h}_{1}\right|^{2}<\left|\tilde{h}_{2}\right|^{2}<\cdots<\left|\tilde{h}_{W}\right|^{2} .
$$

We then turn our attention to the RIS design. It is assumed that the RISs mainly focus on providing maximum channel gain to the prioritized user for enhancing the SE. Without loss of generality, we assume that the prioritized user is the one having the best ordered channel gain.

In this article, in order to simultaneously control multiple RISs, the global CSI is assumed to be perfectly available at

\footnotetext{
${ }^{2}$ A log-normal distributed random variable was considered in [39] for the shadowing of both the desired and interference signals, but it is mathematically intractable, noting that this would not affects the main trends. Thus, we neglected it in this article.
} 
the RIS controller. Since user $W$ is the prioritized user, we aim for maximizing the users' received power by designing the phase shifts and reflection amplitude coefficients of RISs as follows:

$$
\begin{aligned}
& \operatorname{Max}\left(\mathbf{g}_{W} \mathbf{\Phi} \mathbf{h}+r_{W}\right) \\
& \text { subject to } \beta_{1} \cdots \beta_{N}=1 \\
& \theta_{1} \cdots \theta_{N} \in[0,2 \pi) .
\end{aligned}
$$

Thus by utilizing our signal alignment technique, our objective can be achieved by phase-shifting the signals received at the RISs, which is capable of significantly improving the received power. Note that the proposed system outperforms the benchmarks at the additional complexity cost of the RIS. However, the attainable fairness of the network considered is beyond the scope of this treatise.

Thus, we first define a channel vector as follows:

$$
\tilde{\mathbf{h}}=\left[\begin{array}{lll}
g_{W, 1} h_{1} \phi_{1} & \cdots & g_{W, N} h_{N} \phi_{N}
\end{array}\right] .
$$

Hence, the design of the $n$-th RIS can be expressed as

$$
\arg \left(\tilde{h}_{n}\right)=\arg \left(r_{W}\right),
$$

where $\arg (\cdot)$ denotes the angle of the element, and $\tilde{h}_{n}$ denotes the $n$-th element of $\tilde{\mathbf{h}}$.

We then generate the effective vector of user $W$ as follows:

$$
\overline{\mathbf{h}}=\left[\begin{array}{lll}
g_{W, 1} h_{1} & \cdots & g_{W, N} h_{N}
\end{array}\right] .
$$

Thus, the phase shifts of the RISs can be further transformed into

$$
\boldsymbol{\Phi}=\arg (\overline{\mathbf{h}})^{-1} \arg \left(r_{W}\right) .
$$

Since the phase shifts are designed for the prioritized user $W$, the effective channel gain for user $v$ can be written as $\left|\bar{h}_{v}\right|^{2}=\left(\sum_{n=1}^{N} g_{v, n} \theta_{n} h_{n} d_{1}^{-\frac{\alpha_{l}}{2}} d_{2, v}^{-\frac{\alpha_{l}}{2}}+r_{v} d_{3, v}^{-\frac{\alpha_{n}}{2}}\right)^{2}$, which cannot be evaluated. However, for the effective channel gain we have:

$$
0 \leq\left|\bar{h}_{v}\right|^{2} \leq\left|\tilde{h}_{W}\right|^{2}
$$

We then consider the situation that two users, i.e. user $W$ and user $v$ having indexes of $v<W$, are paired to perform NOMA.

\section{B. New Channel Statistics}

In this subsection, we derive new channel statistics for the proposed RIS-aided NOMA network, which will be used for evaluating the OPs and ergodic rates in the following subsections. Table II lists the main symbols used in this article.

Lemma 1. Let us assume that the fading parameters of the elements in $\mathbf{h}$ and $\mathbf{g}_{W}$ are $m_{1}$ and $m_{W}$, respectively. The elements of the channel vectors are independently and identically distributed (i.i.d.). On the one hand, the worst-case distribution of the effective channel gain of user $W$ can be formulated as

$$
\left|\tilde{\mathrm{h}}_{W, l}\right|^{2} \sim \Gamma\left(k_{1}, \lambda_{1}\right),
$$

where $\Gamma(\cdot, \cdot)$ represents the Gamma distribution, and

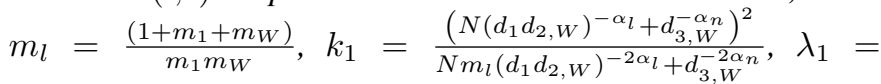

TABLE II:

TABLE OF SYMBOLS

\begin{tabular}{|l|r|}
\hline$\left|\tilde{\mathrm{h}}_{W, l}\right|^{2}$ & The worst-case distribution of user $W$. \\
\hline$\left|\tilde{\mathrm{h}}_{W, u}\right|^{2}$ & The best-case distribution of user $W$. \\
\hline$P_{W, u}$ & The worst-case of the OP expression of user $W$. \\
\hline$P_{W, l}$ & The best-case of the OP expression of user $W$. \\
\hline $\bar{P}_{W, u}$ & The approximated worst-case OP of user $W$. \\
\hline $\bar{P}_{W, l}$ & The approximated best-case OP of user $W$. \\
\hline$d_{W, u}$ & The diversity order of the worst-case of user $W$. \\
\hline$d_{W, l}$ & The diversity order of the best-case of user $W$. \\
\hline$R_{W, l}$ & The worst-case of the ergodic rate of user $W$. \\
\hline$R_{W, u}$ & The best-case of the ergodic rate of user $W$. \\
\hline
\end{tabular}

$\frac{N m_{l}\left(d_{1} d_{2, W}\right)^{-2 \alpha_{l}}+d_{3, W}^{-2 \alpha_{n}}}{N\left(d_{1} d_{2, W}\right)^{-\alpha_{l}}+d_{3, W}^{-\alpha_{n}}}$. On the other hand, the best-case distribution of the effective channel gain of user $W$ can be expressed by

$$
\left|\tilde{\mathrm{h}}_{W, u}\right|^{2} \sim \Gamma\left(k_{2}, \lambda_{2}\right)
$$

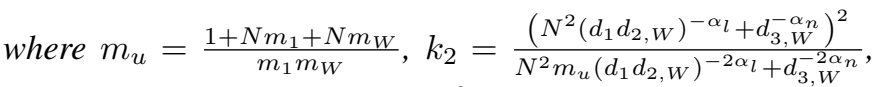
and $\lambda_{2}=\frac{N^{2} m_{u}\left(d_{1} d_{2, W}\right)^{-2 \alpha_{l}}+d_{3, W}^{-2 \alpha_{n}}}{N^{2}\left(d_{1} d_{2, W}\right)^{-\alpha_{l}}+d_{3, W}^{-\alpha_{n}}}$.

Proof: Please refer to Appendix A.

\section{Outage Probability}

In this article, the OP is defined as the probability that the information rate attained is lower than the required target rate. By utilizing SIC, user $W$ first detects the signal of user $v$, and then user $W$ detects its own signal. Hence, the OP of user $W$ is given by

$P_{w}=\mathbb{P}\left(\log _{2}\left(1+S I N R_{W \rightarrow v}\right)<R_{v}\right)+$
$\mathbb{P}\left(\log _{2}\left(1+S I N R_{W \rightarrow v}\right)>R_{v}, \log _{2}\left(1+S I N R_{W}\right)<R_{W}\right)$,

where $R_{W}$ and $R_{v}$ represent the target rates of user $W$ and user $v$, respectively.

We then focus our attention on the SINR analysis of user $W$ having the best channel gain. The cell-centre user $W$ first detects the signal of the cell-edge user $v$ with the following SINR:

$$
S I N R_{W \rightarrow v}=\frac{\left|\tilde{h}_{W}\right|^{2} p \alpha_{v}^{2}}{\left|\tilde{h}_{W}\right|^{2} p \alpha_{W}^{2}+\sigma^{2}} .
$$

Once the signal of user $v$ is detected successfully, user $W$ detects its own signal at an SINR of:

$$
\operatorname{SIN} R_{W}=\frac{\left|\tilde{h}_{W}\right|^{2} p \alpha_{W}^{2}}{\sigma^{2}} .
$$

Let us now turn our attention to calculating the OP of user $W$ based on Theorems 1 and 2 .

Theorem 1. Assuming that $\alpha_{v}^{2}-\alpha_{W}^{2} \varepsilon_{v}>0$, the worst-case of the closed-form OP expression of user $W$ can be expressed 
as

$$
P_{W, u}=\frac{\gamma\left(k_{1}, \frac{I_{w *}}{\lambda_{1}}\right)^{W}}{\Gamma\left(k_{1}\right)^{W}}
$$

where $I_{W *}=\max \left\{I_{w}, I_{v}\right\}, I_{v}=\frac{\varepsilon_{v} \sigma^{2}}{p\left(\alpha_{v}^{2}-\alpha_{W}^{2} \varepsilon_{v}\right)}, I_{W}=\frac{\varepsilon_{W} \sigma^{2}}{p \alpha_{W}^{2}}$, $\varepsilon_{v}=2^{R_{v}}-1, \varepsilon_{W}=2^{R_{W}}-1$, and $\gamma($, ) represents the lower incomplete Gamma function.

Proof: Please refer to Appendix $B$.

Theorem 2. Assuming that $\alpha_{v}^{2}-\alpha_{W}^{2} \varepsilon_{v}>0$, the best-case of the closed-form OP expression of user $W$ can be expressed as

$$
P_{W, l}=\frac{\gamma\left(k_{2}, \frac{I_{w *}}{\lambda_{2}}\right)^{W}}{\Gamma\left(k_{2}\right)^{W}} .
$$

Proof: Similar to Appendix B, Theorem 2 can be readily proved.

It is however quite challenging to directly obtain engineering insights from (20) and (21) due to the $W$-th power of the lower incomplete Gamma function. Thus, in order to gain further insights in the high-SNR regime, the approximate behaviors are analyzed, when the SNR is sufficiently high, i.e. when the transmit SNR obeys $\frac{p}{\sigma^{2}} \rightarrow \infty$.

Corollary 1. Assuming that $\alpha_{v}^{2}-\alpha_{W}^{2} \varepsilon_{v}>0$, the worst-case and best-case of the OP can be approximated in closed form by

$$
\begin{aligned}
\bar{P}_{W, u} & =\left(\sum_{s=0}^{\infty} \frac{1}{\Gamma\left(k_{1}+s+1\right)}\left(\frac{I_{W *}}{\lambda_{1}}\right)^{s}\right)^{W} \\
& \times \sum_{j=0}^{W}\left(\begin{array}{c}
W \\
j
\end{array}\right)(-1)^{j}\left(\frac{I_{W *}}{\lambda_{1}}\right)^{k_{1} W+j},
\end{aligned}
$$

and

$$
\begin{aligned}
\bar{P}_{W, l} & =\left(\sum_{s=0}^{\infty} \frac{1}{\Gamma\left(k_{2}+s+1\right)}\left(\frac{I_{W *}}{\lambda_{2}}\right)^{s}\right)^{W} \\
& \times \sum_{j=0}^{W}\left(\begin{array}{c}
W \\
j
\end{array}\right)(-1)^{j}\left(\frac{I_{W}}{\lambda_{2}}\right)^{k_{2} W+j} .
\end{aligned}
$$

Proof: Please refer to Appendix $C$.

The diversity orders of the prioritized user $W$ can be obtained for evaluating the slope of the OP in the following Propositions.

Proposition 1. Based on Corollary 1, the diversity orders can be determined by using the approximate results. Explicitly the worst-case and best-case of the diversity order of user $W$ supported by the proposed RIS-aided NOMA network are given by

$$
d_{W, u}=-\lim _{\frac{p}{\sigma^{2}} \rightarrow \infty} \frac{\log \bar{P}_{W, u}}{\log \frac{p}{\sigma^{2}}} \approx k_{1} W
$$

and

$$
d_{W, l}=-\lim _{\frac{p}{\sigma^{2}} \rightarrow \infty} \frac{\log \bar{P}_{W, l}}{\log \frac{p}{\sigma^{2}}} \approx k_{2} W .
$$

Remark 1. The results of (24) demonstrate that the diversity orders can be approximated by $\frac{N W}{m_{l}}$ for the prioritized user $W$, when the number of RISs is high enough. It is also demonstrated that increasing the number of RISs and carefully pairing the NOMA users is capable of significantly improving the outage performance.

Remark 2. Assuming that $m_{1} \rightarrow \infty$, which indicates a strong LoS link between the BS as well as the RISs, and provided that the number of RISs is high enough, the diversity orders on both the best-case and worst-case of the prioritized user $W$ can be approximated by $N W m_{W}$.

Remark 3. Since the users are ordered by their effective channel gain, and based on the results of (24), in order to minimize the OP of the paired NOMA users, it is preferable to pair the users having the best and the second best effective channel gains.

Remark 4. Assuming that the number of RISs is high enough, and based on the results of (25), the diversity order of the worst-case on the $O P$ can be further approximated by $\frac{N W m_{1} m_{W}}{m_{1}+m_{W}}$. Again, assuming that $m_{1} \rightarrow \infty$, both the worstcase and best-case on the diversity order of user $W$ can be approximated by $N W m_{W}$, which indicates that the diversity order of both the best-case and worst-case of the OP are identical.

Remark 5. Assuming that the BS-user link of user $W$ is the dominant component, where the path loss exponent $\alpha_{N}=\alpha_{L}$ as well as $d_{1} d_{2, W}>>d_{3, W}$, the diversity order of both the best-case and worst-case are $W$.

Due to the impact of hostile fading environments in $\mathrm{NG}$ networks, it is worth mentioning that no BS-user link may be available between the BS and the paired NOMA users, and the approximate result mainly depends on the 0-th ordered element in (22) and (23). Thus, we continue by providing basic numerical insights using the following Corollary.

Corollary 2. Due to the hostile fading environment between the BS and the users in NG networks, and assuming that $\alpha_{v}^{2}-$ $\alpha_{W}^{2} \varepsilon_{v}>0$, the 0 -th ordered elements in terms of the worstcase and best-case on the approximate OP of user $W$ are given by

$$
\begin{aligned}
\tilde{P}_{W, u} & =\frac{1}{\Gamma\left(\varphi_{1}+1\right)^{W}} \sum_{j=0}^{W}\left(\begin{array}{c}
W \\
j
\end{array}\right)(-1)^{j} \\
& \times\left(\frac{I_{W *}}{m_{l}\left(d_{1} d_{2, W}\right)^{-\alpha_{l}}}\right)^{-\left(\varphi_{1} W+j\right)},
\end{aligned}
$$

and

$$
\begin{aligned}
\tilde{P}_{W, l} & =\frac{1}{\Gamma\left(\varphi_{2}+1\right)^{W}} \sum_{j=0}^{W}\left(\begin{array}{c}
W \\
j
\end{array}\right)(-1)^{j} \\
& \times\left(\frac{I_{W *}}{m_{u}\left(d_{1} d_{2, W}\right)^{-\alpha_{l}}}\right)^{-\left(\varphi_{2} W+j\right)},
\end{aligned}
$$

where $\varphi_{1}=\frac{N}{m_{l}}$, and $\varphi_{2}=\frac{N^{2}}{m_{u}}$.

Remark 6. Assuming that no BS-user links are expected between the BS and the prioritized NOMA user, based on 
results of (26), the best-case and worst-case on the diversity orders of user $W$ are seen to be $\frac{N^{2} W}{m_{u}}$ and $\frac{N W}{m_{l}}$, respectively.

\section{Ergodic Rate}

The ergodic rate is a salient performance metric related to the SE and EE. Therefore, we focus our attention on analyzing the ergodic rate of user $W$. The approximate ergodic rate expressions of user $W$ are given in the following Theorems.

Theorem 3. Assuming that $N$ RISs simultaneously serve user $W$, and $\alpha_{v}^{2}-\alpha_{W}^{2} \varepsilon_{v}>0$, the worst-case on the ergodic rate of user $W$ can be expressed in closed form as follows:

$$
\begin{aligned}
& R_{W, l}=\sum_{s=0}^{W}\left(\begin{array}{c}
W \\
s
\end{array}\right)(-1)^{s} \frac{1}{\bar{k}} \\
& \times \sum_{a_{1}+\ldots+a_{\bar{k}}=s}\left(\begin{array}{c}
s \\
a_{1}, \ldots, a_{\bar{k}}
\end{array}\right) \prod_{t=1}^{\bar{k}}\left(\frac{(C)^{t-1}}{(t-1) !}\right)^{a_{t}} \\
& \times\left(\exp (C s) E i(-C s)+\sum_{i=1}^{(t-1) a_{t}}(-1)^{i-1}(i-1) !(C s)^{i}\right),
\end{aligned}
$$

where $C=\frac{\sigma^{2}}{\lambda_{1} p \alpha_{W}^{2}}$, and $\bar{k}=\left[k_{1}\right]$ is obtained by rounding $k_{1}$ to the nearest integer.

Proof: Please refer to Appendix D.

Similarly, the best-case on the ergodic rate of user $W$ is formulated in the following Theorem.

Theorem 4. Assuming that $N$ RISs simultaneously serve user $W$, and $\alpha_{v}^{2}-\alpha_{W}^{2} \varepsilon_{v}>0$, the best-case on the ergodic rate of user $W$ can be expressed in closed form as follows:

$$
\begin{aligned}
& R_{W, u}=\sum_{s=0}^{W}\left(\begin{array}{c}
W \\
s
\end{array}\right)(-1)^{s} \frac{1}{\bar{k}} \\
& \times \sum_{a_{1}+\ldots+a_{\bar{k}_{u}}=s}\left(\begin{array}{c}
s \\
a_{1}, \ldots, a_{\bar{k}_{u}}
\end{array}\right) \prod_{t=1}^{\bar{k}}\left(\frac{\left(C_{u}\right)^{t-1}}{(t-1) !}\right)^{a_{t}} \\
& \times\left(\exp \left(C_{u} s\right) E i\left(-C_{u} s\right)+\sum_{i=1}^{(t-1) a_{t}}(-1)^{i-1}(i-1) !\left(C_{u} s\right)^{i}\right),
\end{aligned}
$$

where $C_{u}=\frac{\sigma^{2}}{\lambda_{2} p \alpha_{W}^{2}}$, and $\bar{k}_{u}=\left[k_{2}\right]$.

Proof: Similar to Appendix D, the results in (29) can be readily obtained.

To gain deep insights into the system's performance, the high-SNR slope, as the key parameter determining the ergodic rate in the high-SNR regime, is worth estimating. Therefore, we first express the high-SNR slope as

$$
S_{\infty}^{W}=-\lim _{\frac{p}{\sigma^{2}} \rightarrow \infty} \frac{R_{W}}{\log _{2}\left(1+\frac{p}{\sigma^{2}}\right)} .
$$

Proposition 2. By substituting (28) and (29) into (30), the high-SNR slope of user $W$ is given by

$$
S_{\infty}^{W}=1
$$

Remark 7. The results of (31) illustrate that the slope of the ergodic rate in the proposed RIS-aided NOMA network is one, which is not affected by the number of RISs.

Based on the passive beamforming weight design at the RISs, the distribution of NOMA user $v$, having the lower received power, cannot be evaluated. Hence, we only provide the associated SINR analysis for simplicity. By relying on the NOMA protocols, user $v$ treats the signal from user $W$ as interference, and the SINR is given by

$$
S I N R_{v}=\frac{\left(\left|\mathbf{g}_{v} \mathbf{\Phi} \mathbf{h}+r_{v}\right|^{2}\right) \alpha_{v}^{2} p}{\left(\left|\mathbf{g}_{v} \boldsymbol{\Phi} \mathbf{h}+r_{v}\right|^{2}\right) \alpha_{W}^{2} p+\sigma^{2}} .
$$

Since the elements in $\boldsymbol{\Phi}$ are gleaned from random variables, and based on the insights from [35], the SINR of user $v$ can be further approximated as:

$$
\operatorname{SINR}_{v}=\frac{\left(\left|\mathbf{g}_{v} \mathbf{h}\right|^{2} G_{N}(\bar{\theta})+\left|r_{v}\right|^{2}\right) \alpha_{v}^{2} p}{\left(\left|\mathbf{g}_{v} \mathbf{h}\right|^{2} G_{N}(\bar{\theta})+\left|r_{v}\right|^{2}\right) \alpha_{W}^{2} p+\sigma^{2}},
$$

where $G_{N}(\bar{\theta})$ denotes the normalized Fejèr Kernel function with parameter $N$. Note that $G_{N}(\bar{\theta})$ has a period of two, hence $\bar{\theta}$ is uniformly distributed over $[-1,1]$. Thus, the ergodic rate of user $v$ can be expressed as follows.

Theorem 5. Assuming that $N$ RISs simultaneously serve user $W$, and $\alpha_{v}^{2}-\alpha_{W}^{2} \varepsilon_{v}>0$, the worst-case and best-case on the ergodic rate of user $v$ can be expressed as follows:

$$
R_{v, l}=\frac{1}{\ln (2)} \int_{0}^{\frac{\alpha_{v}^{2}}{\alpha_{W}}} \frac{1-F_{v, l}(x)}{1+x} d x,
$$

and

$$
R_{v, u}=\frac{1}{\ln (2)} \int_{0}^{\frac{\alpha_{v}^{2}}{\alpha_{W}^{2}}} \frac{1-F_{v, u}(x)}{1+x} d x,
$$

where $F_{v, l}(x)=\left(\frac{\gamma\left(k_{1}, C_{v, l}\right)}{\Gamma\left(k_{1}\right)}\right)^{v}, F_{v, u}(x)=\left(\frac{\gamma\left(k_{2}, C_{v, u}\right)}{\Gamma\left(k_{2}\right)}\right)^{v}$, $C_{v, l}=\frac{\sigma^{2} x}{\lambda_{1} G_{N}(\bar{\theta}) p\left(\alpha_{v}^{2}-\alpha_{W}^{2} x\right)}$, and $C_{v, u}=\frac{\sigma^{2} x}{\lambda_{2} G_{N}(\bar{\theta}) p\left(\alpha_{v}^{2}-\alpha_{W}^{2} x\right)}$. derived.

Proof: Similar to Appendix $D$, the results can be readily

Remark 8. Let us assume that $\bar{\theta} \rightarrow 0$, indicating that the paired NOMA users share an identical channel vector, then the Fejèr Kernel function can be considered as one. Hence, based on the insights in [41], the best-case and worst-case of the ergodic rate of user $v$ may approach $R_{v}=\log _{2}\left(1+\frac{\alpha_{v}^{2}}{\alpha_{W}^{2}}\right)$ in the high-SNR regime.

In order to provide further insights for RIS-aided NOMA networks, the ergodic rate of the paired users is also analysed in the OMA scenario using TDMA. The OMA benchmark adopted in this article relies on supporting user $W$ and user $v$ in a pair of identical time slots. In each time slot, the RISs provide access only for one of the users. Thus, the channel capacity of user $W$ in the OMA scenario can be expressed as

$$
R_{W, O}=\mathbb{E}\left\{\frac{1}{2} \log _{2}\left(1+S N R_{W, O}\right)\right\}
$$

where $S I N R_{W, O}=\frac{\left(\left(\sum_{n=1}^{N}\left|g_{W, n} h_{n}\right|\right)^{2}\left(d_{1} d_{2, W}\right)^{-\alpha_{l}}+\left|r_{W}\right|^{2} d_{3, W}^{-\alpha_{n}}\right) p}{\sigma^{2}}$. 
Similarly, the channel capacity of user $v$ can be expressed as

$$
R_{v, O}=\mathbb{E}\left\{\frac{1}{2} \log _{2}\left(1+S N R_{v, O}\right)\right\},
$$

where $S I N R_{v, O}=\frac{\left(\left(\sum_{n=1}^{N}\left|g_{v, n} h_{n}\right|\right)^{2}\left(d_{1} d_{2, v}\right)^{-\alpha_{l}}+\left|r_{v}\right|^{2} d_{3, v}^{-\alpha_{n}}\right) p}{\sigma^{2}}$.

\section{E. Spectral Efficiency and Energy Efficiency}

Based on the analysis of the previous two subsections, a tractable SE expression of the proposed RIS-aided NOMA network is given by

$$
S=R_{v}+R_{W} .
$$

In the networks, EE is an important performance metric. Thus, based on insights gleaned from [42], we first model the total power dissipation of the proposed RIS-aided NOMA network as

$$
P_{e}=P_{\mathrm{B}, \mathrm{s}}+2 P_{\mathrm{U}}+p \varepsilon_{b}+N P_{L},
$$

where $P_{\mathrm{B}, \mathrm{s}}$ is the static hardware power consumption of the $\mathrm{BS}, \varepsilon_{b}$ denotes the efficiency of the power amplifier at the BS, $P_{\mathrm{U}}$ is the power consumption of each user, and $P_{L}$ represents the power consumption of each RIS controller. Thus, the EE of the proposed network is given by

$$
\Theta_{E E}=\frac{S}{P_{e}},
$$

where $S$ and $P_{e}$ are obtained from (38) and (39), respectively.

\section{NUMERICAL STUdiES}

In this section, numerical results are provided for the performance evaluation of the proposed network. Monte Carlo simulations are conducted for verifying the accuracy of our analytical results. The bandwidth and carrier frequency of the DL are set to $B W=100 \mathrm{MHz}$ and $1 \mathrm{GHz}$, respectively. The power of the AWGN is set to $\sigma^{2}=-174+10 \log _{10}(B W)$ $\mathrm{dBm}$. The power attenuation at the reference distance is set to $-30 \mathrm{~dB}$, and the reference distance is set to 1 meter. Note that the LoS and NLoS links are indicated by the Nakagami fading parameter, where $m=1$ and $m>1$ are for NLoS and for LoS links, respectively. The target rates are $R_{W}=1.5$ and $R_{v}=1$ bits per channel use (BPCU). The power allocation factors of the paired NOMA users are set to $\alpha_{v}^{2}=0.6$ and $\alpha_{W}^{2}=0.4$. The number of users is set to $W=2$, and $v=1$. The fading environments are set to $m_{1}=m_{W}=m_{v}=1$. The length of the BS-RIS link is set to $d_{1}=60 \mathrm{~m}$. The length of the RIS-user links are set to $d_{2, W}=80 \mathrm{~m}$ and $d_{2, v}=100 \mathrm{~m}$ and those of the BS-user links are set to $d_{3, W}=d_{3, v}=100 \mathrm{~m}$. The path loss exponents of the reflected BS-RIS-user and the direct BS-user links are set to $\alpha_{l}=2.2$ as well as $\alpha_{n}=3.5$, respectively, unless otherwise stated.

1) Impact of the Number of RISs: In Fig. 2, we focus our attention on the OP of the RIS-aided NOMA network. The solid curves and dashed curves represent the worst-case and best-case of the analytical results, respectively. We can see that as the number of RISs serving user $W$ increases, the OP decreases. This is due to the fact that, as more RISs

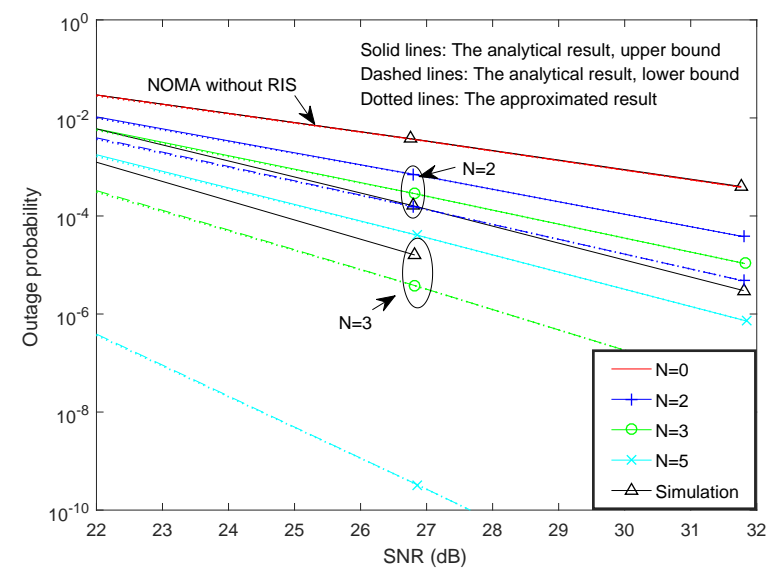

Fig. 2: OP of the RIS-aided NOMA network versus the SNR parameterized by the number of RISs. The analytical and approximate results are calculated from (20), (21), (22) as well as (23), respectively.

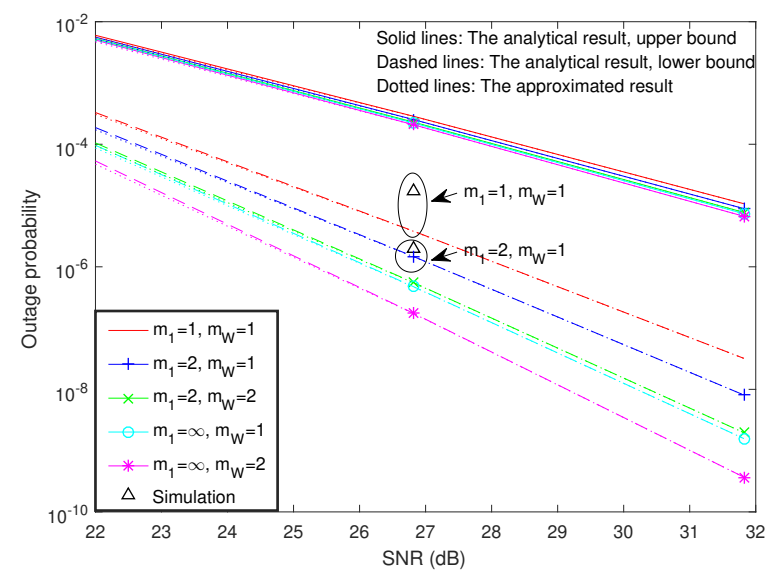

Fig. 3: OP of the RIS-aided NOMA network versus the SNR parameterized by the fading factors. The number of RISs is set to $N=3$.

are employed, the received signal power can be significantly increased as a benefit of the increased diversity order. Observe that the slope of the curves increases with the number of RISs, which validates our Remark 1. Let us assume that $d_{2, W}=d_{3, W}$ and $\alpha_{N}=\alpha_{L}$, then the minimum diversity order that can be obtained is 1 for the case of $m_{1}=m_{W}=1$ and $N=1$, which is identical to that of the non-RISaided networks. Observe that as expected, the simulation results are located between the best and worst cases, which verifies Remark 4.

2) Impact of Fading Environments: In Fig. 3, we evaluate the OP of the prioritized user $W$ in different fading environments. As expected, with the transmit power increases, the OP decreases. Observe that both the BS-RIS as well as RIS-user links have an impact on the OP, which is in contrast to the FOD of [27], where the fading environment of the RIS-user link has almost no effect on the OP.

3) Impact of the Number of Users: Let us now study the impact of the number of users in Fig. 4. Observe that it is preferable to pair the users having the best effective channel 


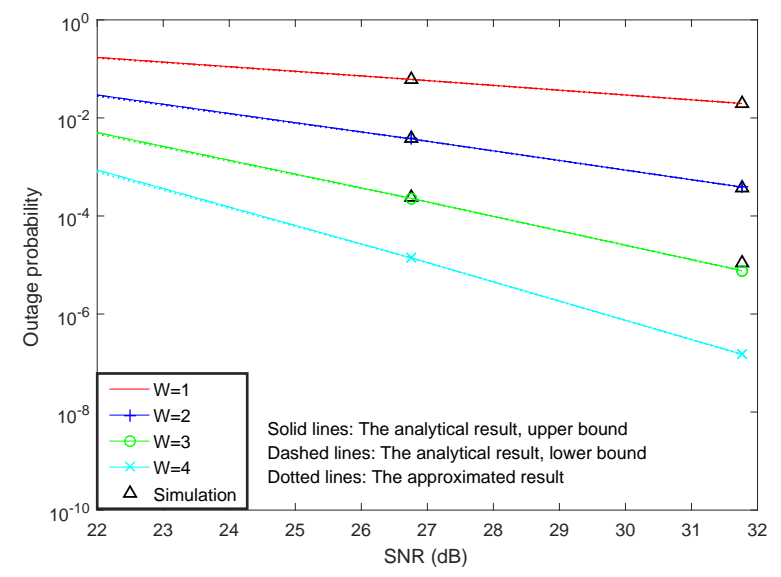

Fig. 4: OP of the RIS-aided NOMA network versus the SNR parameterized by the number of users. The number of RISs is set to $N=3$. The path loss exponents are set to $\alpha_{n}=\alpha_{l}=3$.

gains for minimizing the OP. Based on the results in the highSNR regime, the diversity order is seen to be significantly enhanced by increasing the number of users, because they experience independent fading channels. It is also worth noting that the diversity order is $W$, which is verified by the insights gleaned from Remark 2. This is because when the path loss exponent is $\alpha_{l}=\alpha_{n}=3$, the power received from links reflected by the RISs can be nearly ignored.

4) Ergodic Rate: Fig. 5 compares the ergodic rates of paired NOMA users versus the SNR parameterized by the fading coefficients and by the number of RISs. Several observations can be drawn as follows: 1) Based on the curves in Fig. 5(a), we can observe that the LoS links of both the BS-RIS as well as of the RIS-user links increase the ergodic rate of user $W$, where the ergodic rate approaches the best-case for the case of $m_{1} \rightarrow \infty$. 2) The triangles are between the best-case and worst-case, which verify the accuracy of our results. 3) As seen from the figure, the high-SNR slope of user $W$ is one, which also verifies Remark 8. 4) The ergodic rate can be significantly increased by employing more RISs, which is because the spatial diversity gain can be significantly increased upon increasing the number of RISs. 5) The ergodic rate of conventional NOMA dispensing with RISs is provided as the benchmark schemes, which can be calculated by setting the number of RISs to $N=0.6$ ) Fig. 5(b) evaluates the ergodic rate of the non-prioritized user $v$. Observe that in the highSNR regime, the slope of user $v$ approaches zero in Fig. 5(b), which indicates that the number of RISs has no significant impact on the ergodic rate of user $v$. In TABLE III, we use $\mathrm{D}$ and $\mathrm{S}$ to represent the diversity order and high-SNR slope for the case that $N$ is large enough, respectively. It is worth noting that the diversity order of the non-prioritized user $v$ is the optimized result, which can only be obtained by setting $\bar{\theta} \rightarrow 0$.

5) Comparing the RIS-aided NOMA to an OMA Network: In Fig. 6, we then evaluate the SE of our RIS-aided NOMA network, as well as that of its OMA counterpart. The results of the RIS-aided NOMA and OMA networks are derived by $R_{W}+R_{v}$ and $R_{W, O}+R_{v, O}$, respectively. We can see that

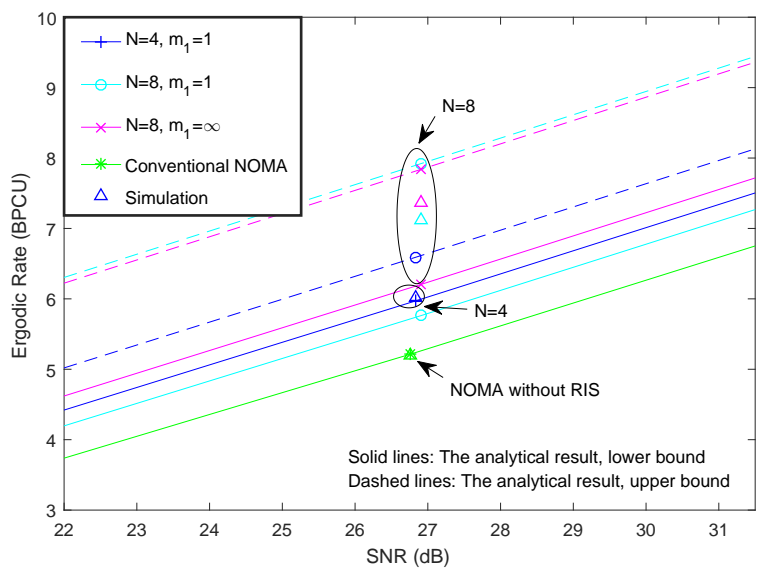

(a) Ergodic rate of user $W$, where the analytical results of user $W$ are calculated from (28) as well as (29).

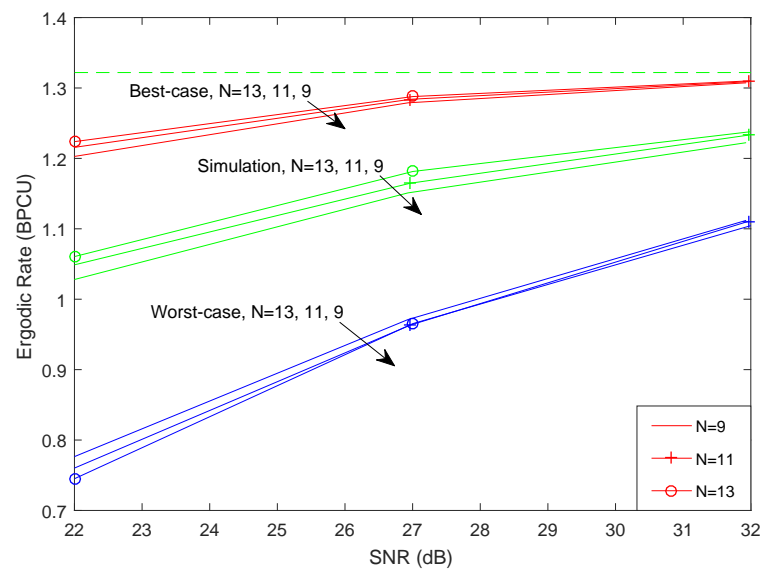

(b) Ergodic rate of user $v$, where the channel gains of the best-case and worst-case are derived similar to (15) and (16).

Fig. 5: Ergodic rate of paired NOMA users versus transmit SNR.

TABLE III:

DIVERSITY ORDER AND HIGH-SNR SLOPE

\begin{tabular}{|l|c|c|c|}
\hline Access Mode & $\mathrm{Rx}$ & $\mathrm{D}$ & $\mathrm{S}$ \\
\hline \multirow{2}{*}{ RIS-aided NOMA } & $W$ & $\frac{N m_{1} m_{W} W}{m_{1}+m_{W}}$ & 1 \\
\cline { 2 - 4 } & $v$ & $\frac{N m_{1} m_{v} v}{m_{1}+m_{v}}$ & 0 \\
\hline \multirow{2}{*}{ Conventional NOMA } & $W$ & $W$ & 1 \\
\cline { 2 - 4 } & $v$ & $v$ & 0 \\
\hline \multirow{2}{*}{ OMA } & $W$ & $W$ & 0.5 \\
\cline { 2 - 4 } & $v$ & $v$ & 0.5 \\
\hline
\end{tabular}

the RIS-aided NOMA network is capable of outperforming its OMA counterpart in terms of its SE by appropriately setting the power allocation factors. Observe that the SE gap between the RIS-aided NOMA network and its OMA counterpart becomes higher, when the number of RISs is increased, which indicates that it is preferable to employ more RISs for enhancing the SE.

6) Comparing the POD to the FOD: In Fig. 7, we evaluate the SE of the proposed POD. The SE of the FOD in [27] is provided as the benchmark. Observe from the figure that the SE of the POD is higher than the FOD of [27], which indicates 


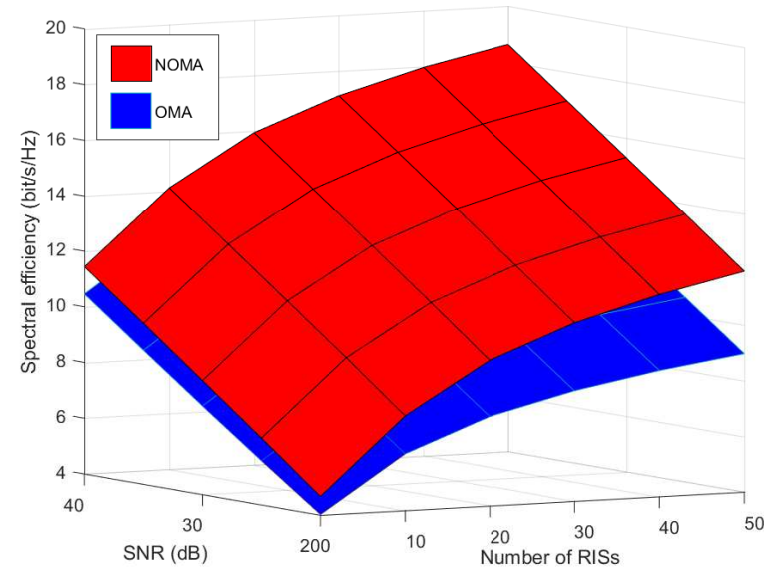

Fig. 6: SE of both the RIS-aided NOMA and OMA networks versus the SNR and the number of RISs. The fading parameters are set to $m_{1}=m_{W}=m_{v}=3$.

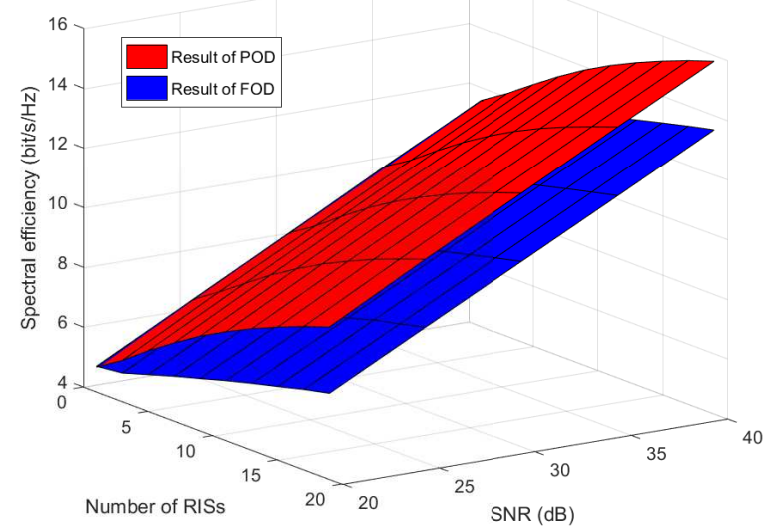

Fig. 7: SE of the proposed RIS-aided NOMA network versus the SNR and the number of RISs. The results of POD and FOD are calculated from (38) and [27]. The fading parameters are set to $m_{1}=m_{W}=m_{v}=3$.

that the proposed POD becomes more competitive compared to the FOD. This is due to the fact that the proposed POD is conceived for attainting the maximum network throughput for the prioritized user, which is capable of providing higher SE. By contrast, the FOD is mainly focused on maintaining fairness, which can provide higher throughput for the cell-edge users.

7) Comparing Half-duplex and Full-duplex Relay Networks:

In order to provide further engineering insights, combined with the insights inferred from [43-45], the network throughputs of alternative full-duplex (FD) and half-duplex (HD) cooperative networks are evaluated. We consider the classic relaying protocols, where the transmissions of HD-relaying are divided into two identical phases. By contrast, the FD-relay is suffering from self-interference. It is assumed that both the BS and relay, as well as the users are equipped by a single antenna. Similar to (7), we first rank the entire set of $W$ users according to their effective channel gains. We then evaluate the SE of the FD network, where the FD-relay has to detect the signal of the paired NOMA users. Based on the insights

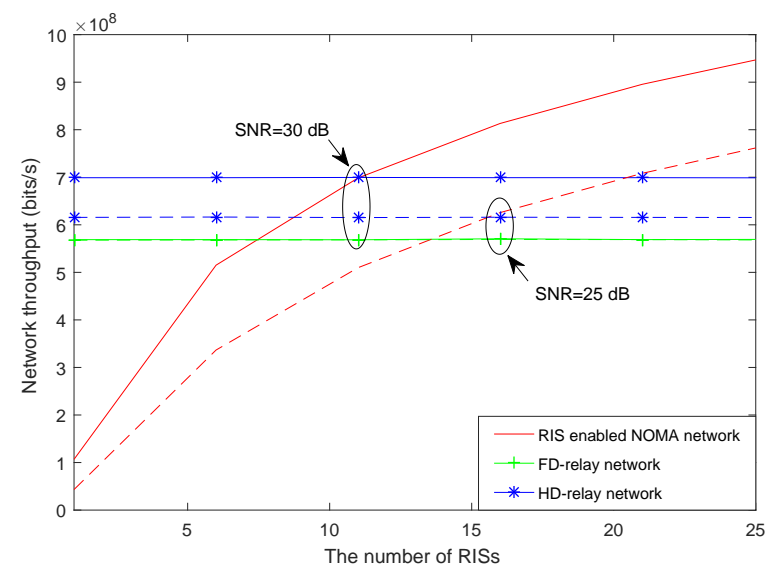

Fig. 8: Network throughput of the RIS-aided NOMA, HDrelay as well as FD-relay networks versus the number of RISs, where the fading parameters are set to $m_{1}=m_{W}=m_{v}=3$. The loop-back self-interference coefficient of FD-relay is set to $\epsilon_{H}=0.01$. The path loss exponent is set to $\alpha_{l}=2.5$.

from Remark 6 for simplicity, it is assumed that no BS-user link exists. Hence, the FD-relay first detects the signal of user $v$, achieving the following expectation:

$$
R_{\mathrm{F}, v}=\mathbb{E}\left\{\log _{2}\left(1+\frac{p\left|h_{R, 1}\right|^{2} d_{1}^{-\alpha_{l}} \alpha_{v}^{2}}{p\left|h_{R, 1}\right|^{2} d_{1}^{-\alpha_{l}} \alpha_{W}^{2}+p_{d} \epsilon_{H}+\sigma^{2}}\right)\right\},
$$

where $\left|h_{R, 1}\right|^{2}$ denotes the channel gain between the BS and the FD-relay, $\epsilon_{H}$ denotes the self-interference coefficient of the FD-relay itself, and $p_{d}$ denotes the transmit power of the relay. Then the FD-relay can detect the signal of user $W$ as follows:

$$
R_{\mathrm{F}, W}=\left\{\log _{2}\left(1+\frac{p\left|h_{R, 1}\right|^{2} d_{1}^{-\alpha_{l}} \alpha_{W}^{2}}{p_{d} \epsilon_{H}+\sigma^{2}}\right)\right\},
$$

Similarly, assuming that SIC can be also invoked successfully by the paired NOMA users, and thus the non-prioritized user $v$ treats the signal of user $W$ as interference, and the expected data rate can be given by

$$
R_{v}=\mathbb{E}\left\{\log _{2}\left(1+\frac{p_{d}\left|h_{R, v}\right|^{2} d_{2, v}^{-\alpha_{l}} \alpha_{v}^{2}}{p_{d}\left|h_{R, v}\right|^{2} d_{2, v}^{-\alpha_{l}} \alpha_{W}^{2}+\sigma^{2}}\right)\right\},
$$

where $\left|h_{R, v}\right|^{2}$ denotes the channel gain between the FD-relay and user $v$. On the other hand, by utilizing SIC technique, the transmission rate of user $W$ is given by

$$
R_{W}=\mathbb{E}\left\{\log _{2}\left(1+\frac{p_{d}\left|h_{R, W}\right|^{2} d_{2, W}^{-\alpha_{l}} \alpha_{W}^{2}}{\sigma^{2}}\right)\right\} .
$$

More specifically, the size of data rate for user $v$ and user $W$ depend on four kinds of data rates, such as 1) the data rate for the relay to detect user $v ; 2$ ) the data rate for the relay to detect user $W$; 3) The data rate for user $v$; and 4) the data rate for user $W$. Based on (41) to (44), the expected rate of the paired NOMA users in the FD-relay network is given by

$$
\bar{R}_{\mathrm{F}, v}=B W \cdot \min \left\{R_{\mathrm{F}, v}, R_{v}\right\},
$$


and

$$
\bar{R}_{\mathrm{F}, W}=B W \cdot \min \left\{R_{\mathrm{F}, W}, R_{W}\right\} .
$$

We then consider the HD-relay network, where the expected data rate of the paired NOMA users at the HD-relay can be given by

$$
R_{\mathrm{H}, v}=\mathbb{E}\left\{\frac{1}{2} \log _{2}\left(1+\frac{p\left|h_{R, 1}\right|^{2} d_{1}^{-\alpha_{l}} \alpha_{v}^{2}}{p\left|h_{R, 1}\right|^{2} d_{1}^{-\alpha_{l}} \alpha_{W}^{2}+\sigma^{2}}\right)\right\},
$$

and

$$
R_{\mathrm{H}, W}=\mathbb{E}\left\{\frac{1}{2} \log _{2}\left(1+\frac{p\left|h_{R, 1}\right|^{2} d_{1}^{-\alpha_{l}} \alpha_{W}^{2}}{\sigma^{2}}\right)\right\} .
$$

By applying the classic SIC technique, the expected data rate of paired NOMA users can be written as

$$
\bar{R}_{v}=\mathbb{E}\left\{\frac{1}{2} \log _{2}\left(1+\frac{p_{d}\left|h_{R, v}\right|^{2} d_{2, v}^{-\alpha_{l}} \alpha_{v}^{2}}{p_{d}\left|h_{R, v}\right|^{2} d_{2, v}^{-\alpha_{l}} \alpha_{W}^{2}+\sigma^{2}}\right)\right\},
$$

and

$$
\bar{R}_{W}=\mathbb{E}\left\{\frac{1}{2} \log _{2}\left(1+\frac{p_{d}\left|h_{R, W}\right|^{2} d_{2, W}^{-\alpha_{l}} \alpha_{W}^{2}}{\sigma^{2}}\right)\right\} .
$$

Thus, the expected rate of the paired NOMA users in the HDrelay network is given by

$$
\bar{R}_{\mathrm{H}, v}=B W \cdot \min \left\{R_{\mathrm{H}, v}, \bar{R}_{v}\right\},
$$

and

$$
\bar{R}_{\mathrm{H}, W}=B W \cdot \min \left\{R_{\mathrm{H}, W}, \bar{R}_{W}\right\} .
$$

In Fig. 8, we evaluate the throughput of our RIS-aided NOMA network, as well as of the HD-relay and FD-relay aided networks. The results of the FD-relay and HD-relay are given by $\bar{R}_{\mathrm{F}, v}+\bar{R}_{\mathrm{F}, W}$ and $\bar{R}_{\mathrm{H}, v}+\bar{R}_{\mathrm{H}, W}$, respectively. The transmit power of the HD-relay and FD-relay are set to $p_{d}=(p-10) \mathrm{dBm}$. We can see that the network throughput gap between the RIS-aided NOMA network and the other pair of relay aided networks becomes smaller, when the number of RISs is increased. Observe that for the case of $N=18$, the proposed RIS-aided NOMA network is capable of outperforming both the FD and HD relay aided networks, which indicates that the RIS-aided NOMA network becomes more competitive, when the number of RISs is high enough.

8) Energy Efficiency: Fig. 9 evaluates the EE of the proposed RIS-aided NOMA network versus the number of RISs. On the one hand, it can be observed that the EE improves as the number of RISs increases. However, observe that the slope of the EE curve is decreasing, which indicates that there exists an optimal value of the number of RISs that maximizes the EE. Furthermore, in contrast to the conventional relay networks [36], it is worth noting that the EE can be increased upon increasing the transmit power at the BS.

\section{CONCLusions}

In this article, we first reviewed the recent advances in RIS-aided networks. In order to illustrate the impact of RISs, we adopted a SISO network, where the passive beamforming weights of the RISs were designed. Both the best-case and

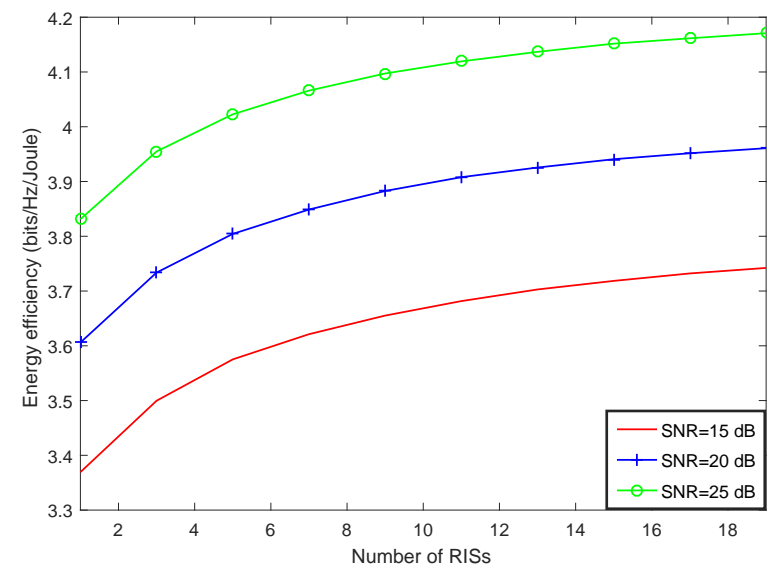

Fig. 9: EE of the proposed RIS-aided NOMA network versus the number of RISs at different SNR. The amplifier efficiency at the BS is set to $\varepsilon_{b}=1.2$. The static power at the BS and users are set to $P_{\mathrm{B}, \mathrm{s}}=9 \mathrm{dBW}$ as well as $P_{\mathrm{U}}=10 \mathrm{dBm}$, respectively. The energy consumption of each RIS is set to $P_{L}=10 \mathrm{dBm}$.

worst-case of our new channel statistics, OPs, ergodic rates, EEs and SEs were derived in closed-form for characterizing the system performance. In NG networks, an important future direction is to extend the proposed model to RIS-aided MIMONOMA networks, where both the active beamforming and passive beamforming, as well as detection vectors can be jointly designed for improving the network's throughput.

\section{Appendix A: Proof of Lemma 1}

In wireless communications, a pair of wave sources may be deemed perfectly coherent if they only have a constant phase difference but the same frequency, as well as the same waveform. In this context, a RIS array exhibits spatial coherence because its elements at the opposite ends of the array have a fixed phase relationship. Therefore, there are two potential scenarios for modeling the channel gain of RIS-aided networks: 1) When the length of the BS-user link and BSRIS-user link is nearly identical, the received signals can be coherently conceived, and thus the channel gain is given by

$$
\left|\tilde{h}_{W}\right|^{2}=\left|\mathbf{g}_{W} \boldsymbol{\Phi} \mathbf{h} d_{1}^{-\frac{\alpha_{l}}{2}} d_{2, W}^{-\frac{\alpha_{l}}{2}}+r_{W} d_{3, W}^{-\frac{\alpha_{n}}{2}}\right|^{2} .
$$

2) On the other hand, since in practice the length of the BSuser and BS-RIS-user links usually varies substantially, the received signals cannot be considered as coherent waves [46]. Thus, when the reflected BS-RIS-user and the direct BS-user signals are co-phased, the BS-user and the BS-RIS-user signals can be boosted at the prioritized user $W$ by utilizing the property of inequations having the following channel gain:

$$
\left|\tilde{h}_{W}\right|^{2} \geq\left|\mathbf{g}_{W} \mathbf{\Phi} \mathbf{h}\right|^{2}\left(d_{1} d_{2, W}\right)^{-\alpha_{l}}+\left|r_{W}\right|^{2} d_{3, W}^{-\alpha_{n}} .
$$

Note that for the first scenario, where the direct BS-user and the reflected BS-RIS-user links are coherent waves, the results of (A.1) can be transformed into (A.2), provided that the number of RISs is high enough. Thus, in the rest of this article, 
we only analyze the network's performance for the second scenario.

Based on the passive beamforming design in (9), the effective channel gain of the prioritized user $W$ can be written as

$$
\left|\tilde{h}_{W}\right|^{2}=\left(\sum_{n=1}^{N}\left|g_{W, n} h_{n}\right|\right)^{2}\left(d_{1} d_{2, W}\right)^{-\alpha_{l}}+\left|r_{W}\right|^{2} d_{3, W}^{-\alpha_{n}} .
$$

By exploiting the fact that the elements of $\left|\mathbf{g}_{W}\right|$ and $|\mathbf{h}|$, as well as of $\left|r_{W}\right|$ are i.i.d., the worst-case of effective channel gain matrix can be transformed into

$$
\begin{aligned}
\left|\tilde{\mathbf{h}}_{W}\right|^{2} & =\sum_{n=1}^{N}\left|g_{W, n} h_{n}\right|^{2}\left(d_{1} d_{2, W}\right)^{-\alpha_{l}}+\left|r_{W}\right|^{2} d_{3, W}^{-\alpha_{n}} \\
& =\sum_{n=1}^{N}\left|g_{W, n}\right|^{2}\left|h_{n}\right|^{2}\left(d_{1} d_{2, W}\right)^{-\alpha_{l}}+\left|r_{W}\right|^{2} d_{3, W}^{-\alpha_{n}}
\end{aligned}
$$

Note that the elements of the channel matrix $\left|\mathbf{g}_{W}\right|$ and $|\mathbf{h}|$ obey the Nakagami distribution having the fading parameters $m_{W}$ and $m_{1}$, respectively. By exploiting the property of random variables, we obtain the mean and variance as follows

$$
E_{1}=\mathbb{E}\left(\left|g_{W, n}\right|^{2}\right) \mathbb{E}\left(\left|h_{n}\right|^{2}\right)=1,
$$

and

$$
\begin{aligned}
& V_{1}=\left(\mathbb{E}\left(\left|g_{W, n}\right|^{2}\right)^{2}+V\left(\left|g_{W, n}\right|^{2}\right)\right) \\
& \times\left(\mathbb{E}\left(\left|h_{n}\right|^{2}\right)^{2}+V\left(\left|h_{n}\right|^{2}\right)\right) \\
& -\mathbb{E}\left(\left|g_{W, n}\right|^{2}\right)^{2} \mathbb{E}\left(\left|h_{n}\right|^{2}\right)^{2}=\frac{\left(1+m_{1}+m_{W}\right)}{m_{1} m_{W}} .
\end{aligned}
$$

Thus, the distribution can be written as

$$
\left|g_{W, n} h_{n}\right|^{2} \sim \mathcal{R} \mathcal{V}\left(1, m_{l}\right)
$$

where $m_{l}=\frac{1+m_{1}+m_{W}}{m_{1} m_{W}}$, and thereby the mean and variance of the effective channel gain is given by

$$
\begin{aligned}
& \sum_{n=1}^{N}\left|g_{W, n} h_{n}\right|^{2}\left(d_{1} d_{2, W}\right)^{-\alpha_{l}} \\
& \sim \mathcal{R} \mathcal{V}\left(N\left(d_{1} d_{2, W}\right)^{-\alpha_{l}}, N m_{l}\left(d_{1} d_{2, W}\right)^{-2 \alpha_{l}}\right) .
\end{aligned}
$$

Due to the fact that user $W$ also detects the signal transmitted from the direct BS-user link, the worst-case of the effective channel gain of user $W$ can be rewritten as

$$
\begin{aligned}
& \left|\tilde{\mathrm{h}}_{W, l}\right|^{2} \sim \mathcal{R} \mathcal{V} \\
& \quad\left(N\left(d_{1} d_{2, W}\right)^{-\alpha_{l}}+d_{3, W}^{-\alpha_{n}}, N m_{l}\left(d_{1} d_{2, W}\right)^{-2 \alpha_{l}}+d_{3, W}^{-2 \alpha_{n}}\right) .
\end{aligned}
$$

After some algebraic manipulations, we obtain the effective channel gain in a more elegant form in (15).

We then turn our attention to the best-case of the effective channel gain of user $W$. By exploiting Cauchy-Schwarz inequality [47], the effective channel gain can be written as

$$
\left(\sum_{n=1}^{N}\left|g_{W, n} h_{n}\right|\right)^{2} \leq\left(\sum_{n=1}^{N}\left|g_{W, n}\right|^{2}\right)\left(\sum_{n=1}^{N}\left|h_{n}\right|^{2}\right) .
$$

Similar to the procedures in (A.5) to (A.9), the best-case of the distribution can be obtained as follows:

$$
\begin{aligned}
& \left|\tilde{\mathrm{h}}_{W, l}\right|^{2} \sim \mathcal{R} \mathcal{V} \\
& \left(N^{2}\left(d_{1} d_{2, W}\right)^{-\alpha_{l}}+d_{3, W}^{-\alpha_{n}}, N^{2} m_{u}\left(d_{1} d_{2, W}\right)^{-2 \alpha_{l}}+d_{3, W}^{-2 \alpha_{n}}\right) .
\end{aligned}
$$

Hence, the best-case distribution can be derived in terms of (16), and the proof is complete.

\section{ApPendiX B: Proof OF THEOREM 1}

Let us first consider the worst-case of the prioritized user $W$. Based on the OP defined in (17), the worst-case of the OP can be rewritten as

$$
P_{W}=\mathbb{P}\left(\left|\tilde{h}_{W, l}\right|^{2}<I_{v}\right)+\mathbb{P}\left(I_{v}<\left|\tilde{h}_{W, l}\right|^{2}<I_{W}\right) .
$$

Since the users are ordered based on their effective channel gain, the marginal PDF of user $W$ is given by [48]

$$
f_{W}(x)=W \tilde{f}_{W}(x)\left(\tilde{F}_{W}(x)\right)^{W-1}
$$

where $\tilde{f}_{W}(x)$ and $\tilde{F}_{W}(x)$ represent the PDF and cumulative density function (CDF) of the unordered effective channel gain associated with $\tilde{f}_{W}(x)=\frac{x^{k_{1}-1}}{\Gamma\left(k_{1}\right) \lambda_{1}^{k_{1}}} \exp \left(-\frac{x}{\lambda_{1}}\right)$ and $\tilde{F}_{W}(x)=$ $1-\frac{\gamma\left(k_{1}, \frac{x}{\lambda_{1}}\right)}{\Gamma\left(k_{1}\right)}$.

Based on the marginal PDF given in (B.2), the OP is given by

$$
P_{W}=W \int_{0}^{I_{W *}} \tilde{f}_{W}(x)\left(\tilde{F}_{W}(x)\right)^{W-1} d x .
$$

After some algebraic manipulations, the OP of the prioritized user $W$ in (20) can be obtained. The proof is complete.

\section{ApPendix C: PROOF OF COROLlary 1}

In order to glean further engineering insights, we first expand the lower incomplete Gamma function as follows [49]:

$$
\begin{aligned}
\gamma\left(k_{1}, \frac{I_{W *}}{\lambda_{1}}\right)= & \sum_{s=0}^{\infty} \frac{\Gamma\left(k_{1}\right)}{\Gamma\left(k_{1}+s+1\right)} \\
& \times\left(\frac{I_{W *}}{\lambda_{1}}\right)^{k_{1}+s} \exp \left(-\frac{I_{W *}}{\lambda_{1}}\right) .
\end{aligned}
$$

In the high-SNR regime, recall that $\lim _{x \rightarrow 0}\left(1-e^{-x}\right) \approx x$. Hence the OP of user $W$ can be approximated in the highSNR regime as follows:

$$
\bar{P}_{W}=\left(\sum_{s=0}^{\infty} \frac{1}{\Gamma\left(k_{1}+s+1\right)}\left(\frac{I_{W *}}{\lambda_{1}}\right)^{k_{1}+s}\left(1-\frac{I_{W *}}{\lambda_{1}}\right)\right)_{(\mathrm{C} .2)}^{W} .
$$

Upon involving the binomial expansion and after some algebraic manipulations, the approximate result can be further 
transformed into

$$
\begin{aligned}
\bar{P}_{W} & =\left(\sum_{s=0}^{\infty} \frac{1}{\Gamma\left(k_{1}+s+1\right)}\left(\frac{I_{W *}}{\lambda_{1}}\right)^{s}\right)^{W} \\
& \times \sum_{j=0}^{W}\left(\begin{array}{c}
W \\
j
\end{array}\right)(-1)^{j}\left(\frac{I_{W *}}{\lambda_{1}}\right)^{k_{1} W+j} .
\end{aligned}
$$

Thus, after some algebraic manipulations, the results in (22) can be obtained, and the proof is complete.

\section{AppendiX D: Proof of Theorem 3}

Let us commence by expressing the worst-case on the ergodic rate of the prioritized user $W$ as follows:

$$
\begin{aligned}
R_{W, l} & =\mathbb{E}\left\{\log _{2}\left[1+S I N R_{W}(x)\right]\right\} \\
& =-\int_{0}^{\infty} \log _{2}(1+x) d[1-F(x)] \\
& =\frac{1}{\ln (2)} \int_{0}^{\infty} \frac{1-F(x)}{1+x} d x .
\end{aligned}
$$

The CDF of user $W$ can be calculated as

$$
F(x)=\left(\frac{\gamma\left(k_{1}, C x\right)}{\Gamma\left(k_{1}\right)}\right)^{W} \text {. }
$$

In order to derive the closed-form expression of the worstcase, we first round the shape parameter to the closest integer, i.e., $\bar{k}=\left[k_{1}\right]$. Hence, the lower incomplete Gamma function can be further expanded to

$$
\frac{\gamma(\bar{k}, C x)}{\Gamma(\bar{k})}=\left(1-\sum_{i=0}^{\bar{k}-1} \frac{(C x)^{i}}{i !} e^{-C x}\right) .
$$

By utilizing the binomial expansion, the result in (D.3) can be further transformed into

$$
\begin{aligned}
& \left(1-\sum_{i=0}^{\bar{k}-1} \frac{(C x)^{i}}{i !} e^{-C x}\right)^{W} \\
& =\sum_{s=0}^{W}\left(\begin{array}{c}
W \\
s
\end{array}\right)(-1)^{s}\left(\sum_{i=0}^{\bar{k}-1} \frac{(C x)^{i}}{i !} e^{-C x}\right)^{s} .
\end{aligned}
$$

Thus, the ergodic rate can be written as

$$
R_{W, l}=\frac{1}{\ln (2)} \sum_{s=1}^{W}\left(\begin{array}{c}
W \\
s
\end{array}\right)(-1)^{s} \int_{0}^{\infty} \frac{e^{-C s x}\left(\sum_{i=0}^{\bar{k}-1} \frac{(C x)^{i}}{i !}\right)^{s}}{1+x} d x .
$$

We then expand (D.5) by using the multi-nomial theorem as follows [50]:

$$
\begin{aligned}
& \left(\sum_{i=0}^{\bar{k}-1} \frac{(C x)^{i}}{i !} e^{-C x}\right)^{s} \\
& =\sum_{a_{1}+\ldots+a_{\bar{k}}=s}\left(\begin{array}{c}
s \\
a_{1}, \ldots, a_{\bar{k}}
\end{array}\right) \prod_{t=1}^{\bar{k}}\left(\frac{(C x)^{t-1}}{(t-1) !}\right)^{a_{t}},
\end{aligned}
$$

where $\left(\begin{array}{c}s \\ a_{1}, \ldots, a_{\bar{k}}\end{array}\right)=\frac{s !}{a_{1} ! \cdot \ldots \cdot a_{\bar{k}} !}$. Thus, the result can be rewritten as

$$
\begin{aligned}
R_{W, l} & =\sum_{s=0}^{W}\left(\begin{array}{c}
W \\
s
\end{array}\right)(-1)^{s} \frac{1}{\bar{k}} \sum_{a_{1}+\ldots+a_{\bar{k}}=s}\left(\begin{array}{c}
s \\
a_{1}, \ldots, a_{\bar{k}}
\end{array}\right) \\
& \times \prod_{t=1}^{\bar{k}}\left(\frac{(C)^{t-1}}{(t-1) !}\right)^{a_{t}} \int_{0}^{\infty} \frac{x^{(t-1) a_{t}} \exp (-C s x)}{1+x} d x .
\end{aligned}
$$

Hence, the tractable approximate results can be derived as

$$
\begin{aligned}
& R_{W, l}=\sum_{s=0}^{W}\left(\begin{array}{c}
W \\
s
\end{array}\right)(-1)^{s} \frac{1}{\bar{k}} \\
& \times \sum_{a_{1}+\ldots+a_{\bar{k}}=s}\left(\begin{array}{c}
s \\
a_{1}, \ldots, a_{\bar{k}}
\end{array}\right) \prod_{t=1}^{\bar{k}}\left(\frac{(C)^{t-1}}{(t-1) !}\right)^{a_{t}} \\
& \times\left(\exp (C s) E i(-C s)+\sum_{i=1}^{(t-1) a_{t}}(-1)^{i-1}(i-1) !(C s)^{i}\right) .
\end{aligned}
$$

Thus, the worst-case on the ergodic rate of user $W$ is obtained in (28), and the proof is complete.

\section{REFERENCES}

[1] T. Hou, Y. Liu, X. Sun, Z. Song, Y. Chen, and A. Li, "NOMA enhanced reconfigurable intelligent surface: A prioritized design," in 2020 IEEE Global Communications Conference: Wireless Communications (Globecom2020 WC), Taipei, China, Dec. 2020.

[2] S. Lien, S. Shieh, Y. Huang, B. Su, Y. Hsu, and H. Wei, "5G new radio: Waveform, frame structure, multiple access, and initial access," IEEE Commun. Mag., vol. 55, no. 6, pp. 64-71, Jun. 2017.

[3] S. Parkvall, E. Dahlman, A. Furuskar, and M. Frenne, "NR: The new 5G radio access technology," IEEE Commun. Standards Mag., vol. 1, no. 4, pp. 24-30, Dec. 2017.

[4] J. Zhang, E. Bjrnson, M. Matthaiou, D. W. K. Ng, H. Yang, and D. J. Love, "Multiple antenna technologies for beyond 5G," Arxiv, vol. 1910.00092, Sep. 2019.

[5] Y. Liang, R. Long, Q. Zhang, J. Chen, H. V. Cheng, and H. Guo, "Large intelligent surface/antennas (LISA): Making reflective radios smart," $J$. Commun. Inf. Networks, vol. 4, no. 2, pp. 40-50, Jun. 2019.

[6] E. Basar, "Transmission through large intelligent surfaces: A new frontier in wireless communications," Arxiv, vol. 1902.08463v2, Apr. 2019.

[7] Z. Ding, Y. Liu, J. Choi, Q. Sun, M. Elkashlan, C. I, and H. V. Poor, "Application of non-orthogonal multiple access in LTE and 5G networks," IEEE Commun. Mag., vol. 55, no. 2, pp. 185-191, Feb. 2017.

[8] Z. Ding, P. Fan, and H. V. Poor, "Impact of user pairing on $5 \mathrm{G}$ nonorthogonal multiple-access downlink transmissions," IEEE Trans. Veh. Technol., vol. 65, no. 8, pp. 6010-6023, Aug. 2016.

[9] M. Shirvanimoghaddam, M. Dohler, and S. J. Johnson, "Massive nonorthogonal multiple access for cellular IoT: Potentials and limitations," IEEE Commun. Mag., vol. 55, no. 9, pp. 55-61, Sep. 2017.

[10] Y. Liu, Z. Qin, M. Elkashlan, Z. Ding, A. Nallanathan, and L. Hanzo, "Nonorthogonal multiple access for 5G and beyond," Proc. of the IEEE, vol. 105, no. 12, pp. 2347-2381, Dec. 2017.

[11] S. M. R. Islam, N. Avazov, O. A. Dobre, and K. Kwak, "Power-domain non-orthogonal multiple access (NOMA) in 5G systems: Potentials and challenges," IEEE Commun. Surveys Tuts., vol. 19, no. 2, pp. 721-742, Secondquarter 2017.

[12] Y. Liu, Z. Qin, M. Elkashlan, A. Nallanathan, and J. A. McCann, "Nonorthogonal multiple access in large-scale heterogeneous networks," IEEE J. Sel. Areas Commun., vol. 35, no. 12, pp. 2667-2680, Dec. 2017.

[13] Q. Wu and R. Zhang, "Towards smart and reconfigurable environment: Intelligent reflecting surface aided wireless network," IEEE Commun. Mag., vol. 58, no. 1, pp. 106-112, 2020.

[14] M. Di Renzo et al., "Smart radio environments empowered by AI reconfigurable meta-surfaces: An idea whose time has come," J. Wireless Commun. Network, vol. 129, 2019. 
[15] M. Di Renzo and J. Song, "Reflection probability in wireless networks with metasurface-coated environmental objects: An approach based on random spatial processes," J. Wireless Commun. Network, vol. 99, 2019.

[16] E. Bjornson, O. Özdogan, and E. G. Larsson, "Intelligent reflecting surface vs. decode-and-forward: How large surfaces are needed to beat relaying?" Arxiv, vol. 1906.03949, Jun. 2019.

[17] C. Huang, G. C. Alexandropoulos, A. Zappone, M. Debbah, and C. Yuen, "Energy efficient multi-user MISO communication using low resolution large intelligent surfaces," in 2018 IEEE GLOBECOM Workshops (GC Wkshps), Dec. 2018, pp. 1-6.

[18] C. Huang, A. Zappone, G. Alexandropoulos, M. Debbah, and C. Yuen, "Reconfigurable intelligent surfaces for energy efficiency in wireless communication," IEEE Trans. Wireless Commun., vol. 18, no. 8, pp. 4157-4170, Aug. 2019.

[19] H. Han, J. Zhao, D. Niyato, M. Di Renzo, and Q. Pham, "Intelligent reflecting surface aided network: Power control for physical-layer broadcasting," arXiv, vol. 1910.14383v1, pp. 1-1, Oct. 2019.

[20] X. Guan, Q. Wu, and R. Zhang, "Intelligent reflecting surface assisted secrecy communication: Is artificial noise helpful or not?" IEEE Wireless Commun. Lett., pp. 1-1, Accepted to appear 2020.

[21] Q. Wu and R. Zhang, "Weighted sum power maximization for intelligent reflecting surface aided SWIPT," arXiv, vol. 1907.05558v2, pp. 1-1, Jul. 2019.

[22] N. S. Perovi, M. Di Renzo, and M. F. Flanagan, "Channel capacity optimization using reconfigurable intelligent surfaces in indoor mmWave environments," arXiv, vol. 1910.14310v1, pp. 1-1, Oct. 2019.

[23] Y. Cao and T. Lv, "Intelligent reflecting surface aided multi-user millimeter-wave communications for coverage enhancement," arXiv, vol. 1910.02398v1, pp. 1-1, Oct. 2019.

[24] G. Zhou, C. Pan, H. Ren, K. Wang, and A. Nallanathan, "Intelligent reflecting surface aided multigroup multicast MISO communication systems," arXiv, vol. 1909.04606v2, pp. 1-1, Sep. 2019.

[25] Q. Wu and R. Zhang, "Beamforming optimization for wireless network aided by intelligent reflecting surface with discrete phase shifts," Arxiv, vol. 1906.03165v2, Jun. 2019.

[26] S. Zhang and R. Zhang, "Capacity characterization for intelligent reflecting surface aided MIMO communication," Arxiv, vol. 1910.13636v1, Oct. 2019

[27] T. Hou, Y. Liu, Z. Song, X. Sun, Y. Chen, and L. Hanzo, "MIMO assisted networks relying on large intelligent surfaces: A stochastic geometry model," arXiv, vol. 1910.00959v1, pp. 1-1, Oct. 2019.

[28] Z. Ding and H. V. Poor, "A simple design of IRS-NOMA transmission," IEEE Commun. Lett., pp. 1-1, Accepted to appear 2020.

[29] X. Mu, Y. Liu, L. Guo, J. Lin, and N. Al-Dhahir, "Exploiting intelligent reflecting surfaces in multi-antenna aided NOMA systems," Arxiv, vol. 1910.13636v1, Oct. 2019

[30] X. Yu, D. Xu, and R. Schober, "MISO wireless communication systems via intelligent reflecting surfaces," Arxiv, vol. 1904.12199v1, Apr. 2019.

[31] M. Fu, Y. Zhou, and Y. Shi, "Intelligent reflecting surface for downlink non-orthogonal multiple access networks," arXiv, vol. 1906.09434v3, pp. 1-1, Jun. 2019.

[32] E. Basar, "Large intelligent surface-based index modulation: A new beyond MIMO paradigm for 6G," Arxiv, vol. 1904.06704v1, Apr. 2019.

[33] G. Yang, X. Xu, and Y. Liang, "Intelligent reflecting surface assisted non-orthogonal multiple access," Arxiv, vol. 1907.03133v1, Jul. 2019.

[34] A. Goldsmith, Wireless Communication. Cambridge University Press, 2nd ed, 2010.

[35] W. Yi, Y. Liu, A. Nallanathan, and M. Elkashlan, "Clustered millimeterwave networks with non-orthogonal multiple access," IEEE Trans. Commun., vol. 67, no. 6, pp. 4350-4364, Jun. 2019.

[36] K. Ntontin, J. Song, and M. Di Renzo, "Multi-antenna relaying and reconfigurable intelligent surfaces: End-to-end SNR and achievable rate," Arxiv, vol. 1908.07967v2, Aug. 2019.

[37] F. P. Fontàn and P. M. Espinẽira, Modelling the wireless propagation channel: A simulation approach with MATLAB. John Wiley, 2008.

[38] L. Dai, B. Wang, M. Wang, X. Yang, J. Tan, S. Bi, S. Xu, F. Yang, Z. Chen, M. Di Renzo, and L. Hanzo, "Reconfigurable intelligent surface-based wireless communications: Antenna design, prototyping, and experimental results," IEEE Access, vol. 8, pp. 45 913-45 923, 2020.

[39] J. G. Andrews, F. Baccelli, and R. K. Ganti, "A tractable approach to coverage and rate in cellular networks," IEEE Trans. Commun., vol. 59, no. 11, pp. 3122-3134, Nov. 2011.

[40] T. Hou, X. Sun, and Z. Song, "Outage performance for non-orthogonal multiple access with fixed power allocation over Nakagami- $m$ fading channels,' IEEE Commun. Lett., vol. 22, no. 4, pp. 744-747, Apr. 2018.
[41] T. Hou, Y. Liu, Z. Song, X. Sun, and Y. Chen, "Multiple antenna aided NOMA in UAV networks: A stochastic geometry approach," IEEE Trans. Commun., vol. 67, no. 2, pp. 1031-1044, Feb. 2019.

[42] L. N. Ribeiro, S. Schwarz, M. Rupp, and A. L. F. de Almeida, "Energy efficiency of mmWave massive MIMO precoding with low-resolution DACs," IEEE J. Sel. Topics Signal Process, vol. 12, no. 2, pp. 298-312, May 2018.

[43] Y. Liu, Z. Ding, M. Elkashlan, and H. V. Poor, "Cooperative nonorthogonal multiple access with simultaneous wireless information and power transfer," IEEE J. Sel. Areas Commun., vol. 34, no. 4, pp. 938953, Apr. 2016.

[44] J. N. Laneman, D. N. C. Tse, and G. W. Wornell, "Cooperative diversity in wireless networks: Efficient protocols and outage behavior," IEEE Trans. Information Theory, vol. 50, no. 12, pp. 3062-3080, Dec. 2004.

[45] X. Yue, Y. Liu, S. Kang, A. Nallanathan, and Z. Ding, "Spatially random relay selection for full/half-duplex cooperative NOMA networks," IEEE Trans. Commun., vol. 66, no. 8, pp. 3294-3308, Aug. 2018.

[46] R. G. Winter and A. M. Steinberg, Coherence. Access Science. McGraw-Hill., 2008.

[47] Z. Zhao, Z. Ding, M. Peng, and Y. Li, "A full-cooperative diversity beamformingscheme in two-way amplify-and-forward relay systems," Digital Communications and Networks, vol. 1, no. 1, pp. 57-67, Mar. 2015.

[48] H. N. Nagaraja and H. A. David, Order Statistics. John Wiley, New York, 3rd ed, 2003.

[49] I. S. Gradshteyn and I. M. Ryzhik, Table of Integrals, Series and Products. New York: Academic Press, 6th ed, 2000.

[50] N. Bourbaki, Elements of the History of Mathematics Paperback. Springer Berlin Heidelberg, 2nd ed, 2008.

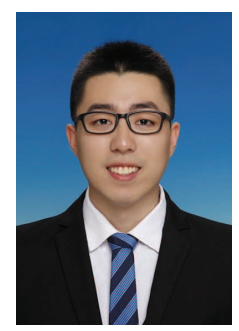

Tianwei Hou received the M.Sc. degrees from Beijing Jiaotong University, and is currently working toward the Ph.D. degree in the School of Electronic and Information Engineering at Beijing Jiaotong University, all in Information and Communication Engineering. His research interests include $5 \mathrm{G}$ networks, wireless communications theory, nonorthogonal multiple access, cooperative networks and Internet of Things.

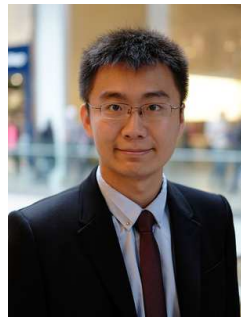

Yuanwei Liu (S13-M16) received the B.S. and M.S degrees from the Beijing University of Posts and Telecommunications in 2011 and 2014, respectively, and the $\mathrm{Ph} . \mathrm{D}$. degree in electrical engineering from the Queen Mary University of London, U.K., in 2016. He was with the Department of Informatics, Kings College London, from 2016 to 2017, where he was a Post-Doctoral Research Fellow. He has been a Lecturer (Assistant Professor) with the School of Electronic Engineering and Computer Science, Queen Mary University of London, since 2017.

His research interests include $5 \mathrm{G}$ wireless networks, Internet of Things, machine learning, stochastic geometry, and matching theory. He received the Exemplary Reviewer Certificate of the IEEE WIRELESS COMMUNICATION LETTERS in 2015, the IEEE TRANSACTIONS ON COMMUNICATIONS in 2016 and 2017, the IEEE TRANSACTIONS ON WIRELESS COMMUNICATIONS in 2017. Currently, $\mathrm{He}$ is in the editorial board of serving as an Editor of the IEEE COMMUNICATION LETTERS and the IEEE ACCESS. He also serves as a guest editor for IEEE JSTSP SPECIAL ISSUE ON "SIGNAL Processing Advances for Non-Orthogonal Multiple Access in NeXt Generation Wireless Networks". He has served as the Publicity Co-Chairs for VTC2019-FALL. He has served as a TPC Member for many IEEE conferences, such as GLOBECOM and ICC. 


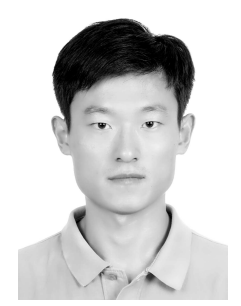

Zhengyu Song received the B.Sc. and M.Sc. degrees from Beijing Jiaotong University and the Ph.D. degree from Beijing Institute of Technology, Beijing, China, all in Information and Communication Engineering. He is currently with the School of Electronic and Information Engineering, Beijing Jiaotong University, Beijing, China. His research interests lie in cooperative communications and radio resource management in multi-carrier systems.

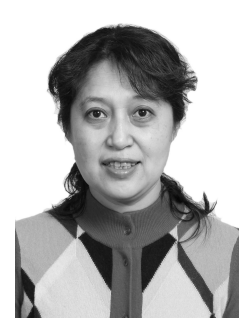

Xin Sun received the Ph.D. degree in electromagnetic measurement technology and instrument from Harbin Institute of Technology, Harbin, China. She is currently a Professor with the School of Electronic and Information Engineering, Beijing Jiaotong University, Beijing, China. Her main research interests are professional mobile communications and wireless personal communications.

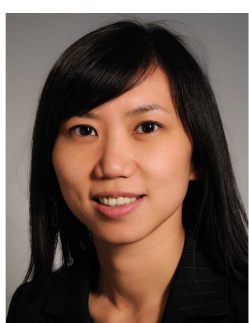

Yue Chen (S02-M03-SM15) received the bachelors and masters degrees from the Beijing University of Posts and Telecommunications, Beijing, China, in 1997 and 2000, respectively, and the Ph.D. degree from the Queen Mary University of London (QMUL), London, U.K., in 2003. She is currently a Professor of telecommunications engineering with the School of Electronic Engineering and Computer Science, QMUL. Her current research interests include intelligent radio resource management for wireless networks, cognitive and cooperative wireless networking, mobile edge computing, HetNets, smart energy systems, and Internet of Things. She is in the editorial board of serving as an Editor of the IEEE COMMUNICATION LETTERS. She has served as a TPC Member for many IEEE conferences, such as GLOBECOM and ICC.

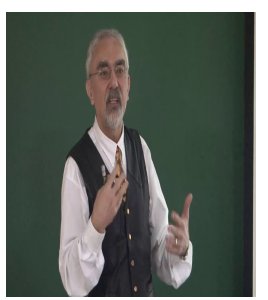

Lajos Hanzo (http://www-mobile.ecs.soton.ac.uk, https://en.wikipedia.org/wiki/Lajos_Hanzo) (FIEEE'04, Fellow of the Royal Academy of Engineering F(REng), of the IET and of EURASIP), received his Master degree and Doctorate in 1976 and 1983, respectively from the Technical University (TU) of Budapest. He was also awarded the Doctor of Sciences (DSc) degree by the University of Southampton (2004) and Honorary Doctorates by the TU of Budapest (2009) and by the University of Edinburgh (2015). He is a Foreign Member of the Hungarian Academy of Sciences and a former Editor-in-Chief of the IEEE Press. He has served several terms as Governor of both IEEE ComSoc and of VTS. He has published 1900+ contributions at IEEE Xplore, 19 Wiley-IEEE Press books and has helped the fast-track career of $123 \mathrm{PhD}$ students. Over 40 of them are Professors at various stages of their careers in academia and many of them are leading scientists in the wireless industry. 